\title{
Characterization of Nano-scale Instabilities in Titanium Alloys Using Aberration- Corrected Scanning Transmission Electron Microscope
}

\author{
Yufeng Zheng ${ }^{1}$, Robert E.A. Williams ${ }^{1}$, Hamish L. Fraser ${ }^{1}$
}

${ }^{1}$ Center for the Accelerated Maturation of Materials and Department of Materials Science and Engineering, The Ohio State University, Columbus, OH, USA.

Due to the refined nature of microstructures that can be effectively manipulated by the application of various thermal/mechanical processes, metastable beta titanium alloys have attracted considerable attention in recent days. Usually, such refinement involves the precipitation of the intragranular $h c p$ structure alpha phase. In authors recent studies, it has been shown that the size, morphology and number density of these alpha precipitates in Ti-5Al-5Mo-5V-3Cr (Ti-5553, wt.\%) can be significantly influenced by the nano-scale structural and compositional instability present in parent $b c c$ structure beta phase, more specifically in this alloy, the metastable hexagonal structure isothermal omega phase [1-3]. In these latter studies, it was found that either the compositional and/or stress field associated with the isothermal omega phase may contribute to an increased driving force for alpha nucleation [3]. Recent technological improvement in TEM's, spectroscopy detectors and cameras, specifically probe aberration corrected STEM instruments, have enabled atomic resolution z-contrast high angle annular dark fieldscanning transmission electron microscopy (HAADF-STEM) imaging capable of characterizing atomic column configurations with a sub-angstrom probe [4] and provides novel insights of new nano-scale instabilities in titanium alloys.

In the first part of this current work, the crystallography and the structure of a previous unidentified ordered orthorhombic metastable phase, termed as O" phase, in the beta matrix of Ti-5553 were studied using probe corrected scanning transmission electron microscope (FEI Titan ${ }^{\mathrm{TM}}$ 80-300) [5]. For the first time, using Z-contrast HAADF-STEM imaging, a novel ordering mechanism in beta titanium alloy, that one of every three $\{110\}$ beta planes exhibits a relative lower intensity than the other two as shown in Fig 1(a), is clearly observed and validated. The lattice parameters of O" phase are $a=0.328 \mathrm{~nm}$, $b=0.464 \mathrm{~nm}$ and $c=1.393 \mathrm{~nm}$, with $\alpha=\beta=\gamma=90^{\circ}$ and $\mathrm{O}$ " phase obeys the following OR with the parent $\beta$

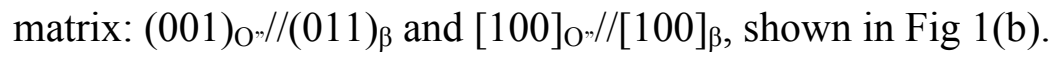

The second part of the work to be presented focused on the characterization of a newly characterized nano-scale sturcutual instability in $\mathrm{Zr}$ added $\mathrm{Ti}-\mathrm{Nb}$ beta type titanium alloy [6]. This new phase, termed as $0^{\prime}$ phase, was investigated using probe corrected scanning transmission electron microscope (FEI Titan $^{\mathrm{TM}}$ 80-300). A high magnification HAADF image is shown in Fig. 2(a), in which it is clear that in the lower left part of the image, bounded by the dashed red box, the $b c c$ symmetry has been broken by small displacements of every other atomic column. These displacements result in a structure very similar to that shown in the schematic diagram in Fig. 2(b), which depicts the result of the application of the $\{110\}<1 \overline{1} 0>$ shuffle, i.e., a disordered orthorhombic structure.

\section{References:}

[1] Y. Zheng et al, Scripta Materialia, 111 (2016), p. 81.

[2] Y. Zheng et al, Acta Materialia, 103 (2016), p. 165.

[3] Y. Zheng et al, Acta Materialia, 103 (2016), p. 850. 
[4] H.L. Fraser et al, Transmission Electron Microscopy for Physical Metallurgists Elsevier Science, Burlington, 2014.

[5] Y. Zheng et al, Scripta Materialia, 113 (2016), p. 202.

[6] Y. Zheng et al, Scripta Materialia, 116 (2016), p. 49.

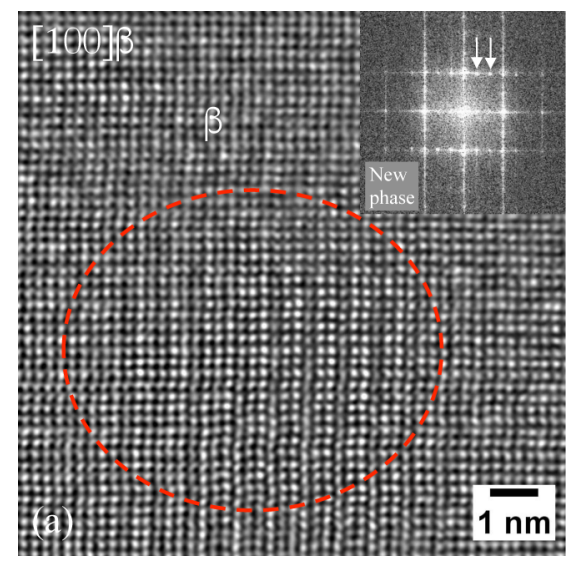

(b)
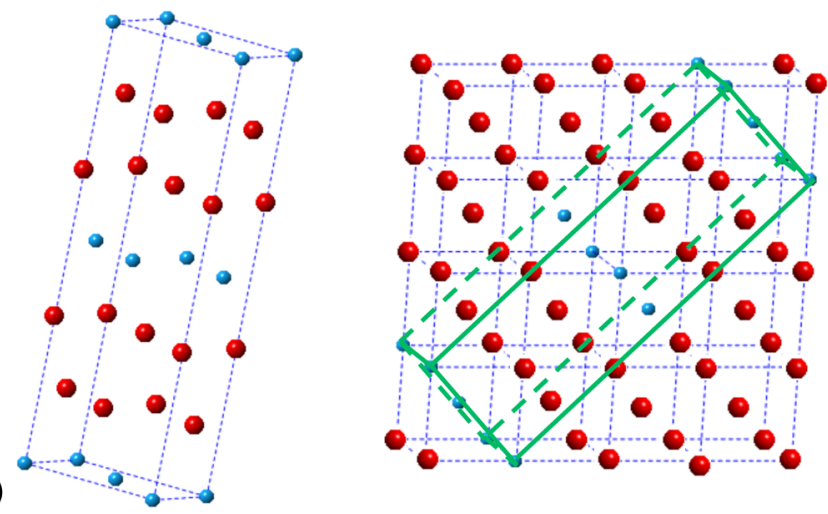

Figure 1. (a) Z-contrast HAAD-STEM image showing the nano-scale ordered orthorhombic structure $\mathrm{O}^{\prime \prime}$ phase; (b) schematic figure showing the structure of $\mathrm{O}^{\prime \prime}$ phase and its orientation relationship with beta phase matrix [5].
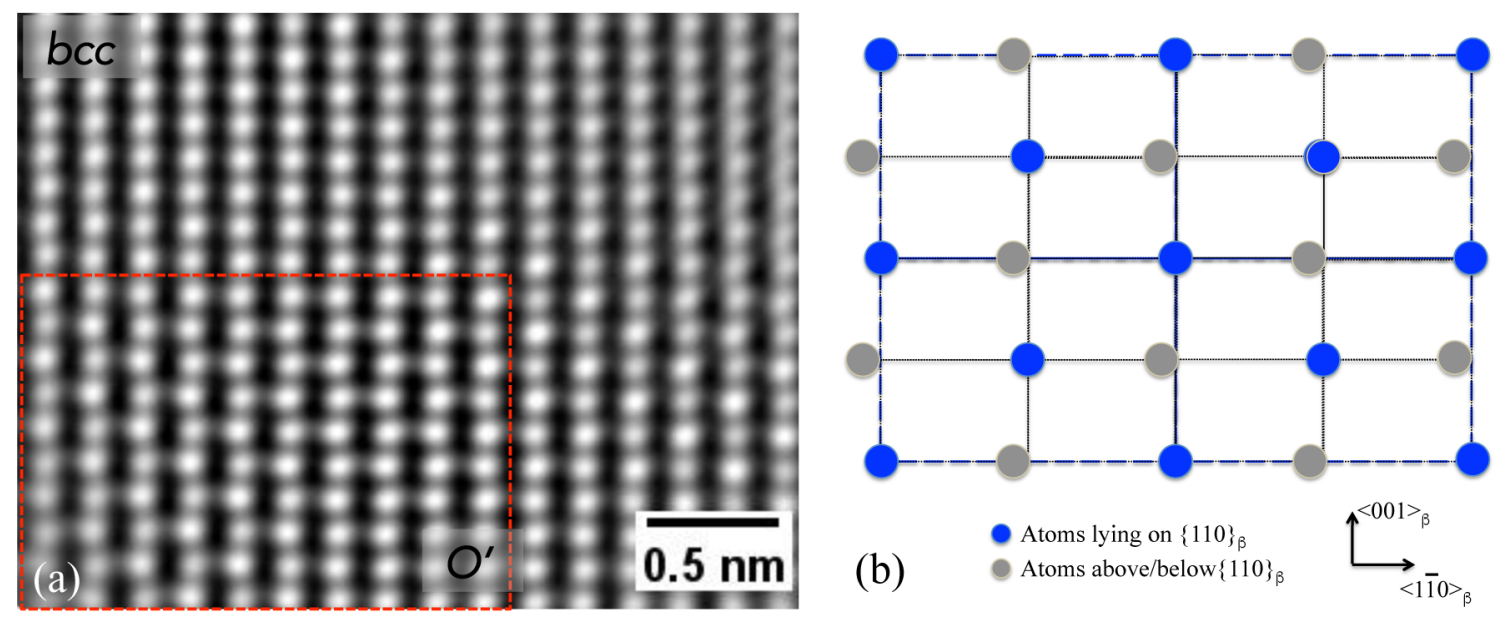

Figure 2. (a) Z-contrast HAAD-STEM image showing the nano-scale disordered orthorhombic structure $\mathrm{O}^{\prime}$ phase; (b) schematic figure showing the structure of $\mathrm{O}^{\prime}$ phase [6]. 